\title{
Research for Two-way Real-name Authentication Unified Platform of C2C E-commerce Based on DNS
}

\author{
Xiaotang Li \\ Computer and Information Engineering College \\ Harbin University of Commerce \\ Harbin, China \\ htyiguo@qq.com
}

\begin{abstract}
C2C e-commerce credit problems is the root of the problems, and its essence is that the mastering for user true information is not enough. It is proposed in this paper, twoway real-name authentication platform based on DNS, the platform for buyers and sellers make unified authentication to strengthen the management of the buyer, by the user number and identity information of the binding to defend the credibility of user number, at the same time, by user number realizing the trading online by single number integrates user's credibility on different sites, guarantees the reliability of the user credit information. According to the various characteristics of the platform implementation technology, this paper puts forward a two-way authentication unified platform chosen the DNS system as the foundation, it can reduce the difficulty of the implementation technology, also decrease the demand for each site.
\end{abstract}

Keywords: two-way real-name authentication platform based on DNS, unified authentication, user number

\section{Necessity of Two-way Real-name Authentication}

The credit problems are derived from the asymmetry of credit about buyer and seller in $\mathrm{C} 2 \mathrm{C}$ e-commerce, the asymmetric credit is mainly embodied in two aspects: commodity information asymmetry and transaction both sides information asymmetry, namely that the buyer can master seller's information certainly from a website, and the seller to the buyer's information to grasp very few, and little is known about commodity information to the buyer but the seller masters entirely. Therefore, caused many credit problems in C2C transactions, for example: credit hype, fraudulent trading, etc. The key to solve the problem of asymmetric credit is two-way real-name certification, ensure the buyer and the seller equal treatment in the e-commerce market.

\subsection{Credit Hype}

For buying and selling both sides in order to raise the credit for the purpose, or in the case of no actual transaction both sides make a high praise, is the credit hype. Because in every e-commerce website real-name certification, is only for the seller but for the buyer only have part of a certification of ability to pay during payment, is relatively easy. For seller of real-name authentication only limits the same identity $i$ in many times $n$ the registered a web site, but does not rule out the same seller can be registered as a buyer, and by the behavior of trading with himself conducts credit hype. The way of Credit hype includes: trading with himself, paying to work team hype credit, free in the group of mutual credit hype, brush drilling platform and the use of automatic prepaid system to enhance credit and so on. Credit hype is very dangerous for the network market speculation, the specific performance as follows [1]: 
(1) Improve the market access costs. Company providing credit hype will have service fee, so there is no doubt that will increase market access costs, and increase the costs of market integrity at the same time;

(2) Create network market bubble. In the network credit speculation behavior once prosperity will cause network trading illusion of prosperity. Due to network monetary liquidity is strong, very small amounts of money through a control network can generate high market transaction volume. Simply put real money into the network market is very little, but by buying and selling each other, the network market is big, this will give some wrong decision organization, so as to make some wrong decisions.

(3) The shop owner risk is big. Credit hype often only one or several operations, of course not rule out such network organization. But, as long as the impacts to the benefit of the web site, web site only have a little change about assessment mechanism, the owner is entirely possible exposure;

(4) The supplier is not reliable credit. To provide such services, often need money before service.

\subsection{Fraudulent Trading}

As well as problems in credit hype, each site limit the seller in the same register only once, but does not limit the identity of the user in different registered in the same site many times, more can't restrict users registered in different sites, so brings the convenience for the user doing fraudulent trading. Fraudulent transactions also calls the counterfeiting, namely that operators by fake, counterfeit, or other fraudulent means, make trading counterpart confused or mistaken belief, thereby gaining trading opportunities. Fraudulent transactions include four kinds of specific performance [2]:

(1) Fake the registered trademark of others, this kind of behaviour is refers to the behaviour no permission of the trademark owner to use its registered trademark, violate the trademark owner rights to exclusive use of trademarks, at the same time constitute behaviour of unfair competition.

(2) The behaviour of confuse with well-known goods. It refers to the unauthorized use of the specific name, packaging, decoration of well-known goods or using with the wellknown goods similar name, package and decoration, and result confused others wellknown trademark, make the buyer's mistake knowing behaviour.

(3) Unauthorized use of the enterprise name or the name of others, make people mistaken for the goods of others, this kind of behaviour is falsely use another producer name or the name of the enterprise make the enterprise's competitive advantage is weakened.

(4) Forged, fraudulent behaviour of various kinds of quality marks and producing area. Including product quality certification, producing area, etc.

\section{Two-way Real-name Authentication Unified Platform Model Based on DNS}

\subsection{Unified Platform Design Characteristics of the Two-way Real-name Authentication}

Two-way real-name authentication unified platform for all sites provides user authentication platform, design features of the platform below [3]:

(1) Users of unity. Namely that the authentication users on this two-way real-name authentication unified platform do not distinguish between the buyer or the seller, as long as is certificated in this platform he will have the user's identity.

(2) Certification of unity. Only authentication users in this platform will get a number, with the user number can be traded in various sites. 
(3) Certification of the system. In the platform authentication uses real identity information, certification id information can only be certified one time, and get a unique user number for online transactions. (Suggestion: the server should get id card information from the public security network.)

(4) The unity of site management. That the platform can implement unified management of credit information to all site users. (5) Based on DNS. That is, the platform is based on DNS system.

\subsection{Implementation Technology of the Two-way Real-name Authentication Unified Platform}

Two-way real-name authentication needs to have a unified platform of each site public platform to user information and registration information for each site for unified management, consider developing a with functions of this website can't just rely on the power of the society, more important is the need for a national management attitude, and the site can't belong to any social business website, cause embodied in the following points:

(1) Because the platform authentication must be absolutely true, fair and reasonable, any commercial website don't have such requirement;

(2) Get the platform development and management of commercial websites will gain profits, for example: originally just a website user login the website, and get the two-way real-name authentication unified platform for the development and management, the website login user will be all the Chinese web site users to deal, so will cause imbalance of the market [4];

(3) Two-way real-name authentication user Numbers of unified platform is massive, requirements for the performance of the system is very high, in general the server can't meet the requirements [6].

Based on these reasons, the designs of unified platform is established on the basis of the DNS server, relying on the DNS technology, the advantage, reduces the risk of the platform development and reduce the difficulty of the implementation technology, it realizes the feasibility of management at the same time. The technical characteristics of implementation of Two-way real-name authentication unified platform are as follows:

(1) No independent website for management of this certification, but relies on the management of DNS. By the user in the first transaction to register on a website, both the buyer and the seller, as long as to trade on the website will be have a real-name authentication, the authentication is through the web server's domain management server. After real-name certification for a user, he will get a user number, the user number and real name information are bound together, are kept in the DNS server, then when the user trades again no matter in which website, as long as the trade will be have a real-name authentication, if there are already this user Numbers on DNS server, he will not be allowed to register, namely that the user can only use his online trading for the first time in a website to register to do online trading. DNS for technical basis because DNS is a structured management system, such a technology base, completely accords with the management characteristics of the platform, solves the problem of development technology and platform management problems.

(2) In this platform the web serve IP when registering for the first time will be look as the identification for DNS system, namely whether buy party or the seller, whether setting up shop or transactions, as long as register will ever with the web server to connect to the server's domain management server for real-name authentication and get a user, the user number is the pass card trading on net for real-name user. If have already generated user number information in the DNS server, then to register is invalid. Do the benefits of each web site can be unified, avoid the users from doing fraudulent trading on different sites as the same real name, and avoid the same user from registering on the deferent sites in different status , and thus for the buys from selling behavior to do credit hype. 
(3) Each site processes credit management by the user number. Such management based on one number is built on a base with real-name information binding, so it is feasible. And the management based on one number can make the trading credit uniform while the user trades online, every trading user in site need to be registered, if he has registered it will not go the registration process, but generates the current trading web IP information to bind with the real-name user information, will be managed in the DNS server, but if the user has been registered in this website that trading will not repeat binding the user number, real name information and the website IP. So if you want to query the user's credit information you can through its unique user number to the DNS server access to get all his trading website IP, and then can query all credit information of the user to the relevant sites;

(4) A child Table will be added to all levels of the DNS server IP and domain Table, to record all users information based on this IP .This technology does not need to do much change to existing DNS system, it can simplify the development process.

\subsection{Model Design}

Through the model chart 1 and chart 2 below to explain the user registration process trade on the net for the first time of the bidirectional real-name authentication unified platform (see Figure1 and Figure2):

\section{Figure 1. Model Chart One}

(Note: the user registration information includes identity card number, user number, the first registered website server IP)

\section{Figure 2. Model Chart Two}

The user in this platform is divided into two types, one is to trade on the net for the first time, this kind of user needs to register when he is trading in site, through the website to be connect to the DNS server the web server IP belonging to, in the DNS server for registration, registration certification through id information, if the user id information database is queried to have be existing, the user will not be registered, otherwise he will be registered and generate user registration information stored in the user information database, in the model the management and query of user information database are based 
on the layered management for DNS system, namely if the DNS query less than it will apply next higher level for the service (see Figure3) [6], Of course, the premise of the query is website domain name resolution, domain name resolution process will not be introduced here. So can uniform each website user management, which coincides with the DNS system management thought, after registration the DNS server the generated user number will be passed to the user's web site, through the web site passed to the user. Another user is the user non first time in online trading, the user has had an user number though his first time trading, can be directly by the user number to trade without having to register online, but when the user input user number on the website, web site needs to the DNS server authentication, after the DNS query authentication will return a confirmation, that is the user number is existing and only put the website IP into the user information database, binding with the user's information, if the bindings have been made on the site's IP will no longer be repeated binding. Maybe also there is registered users with wrong operation, which has been registered but due to forget or unclear trading process has carried on the registration, the process is consistent with the second user type, passed through the website to the DNS server is registration information, through the DNS query this user is no longer accepting and return confirmation notice. Whatever the user can through the user number to query DNS server yourself and others deal website IP, thus query user credit information in each transaction website.

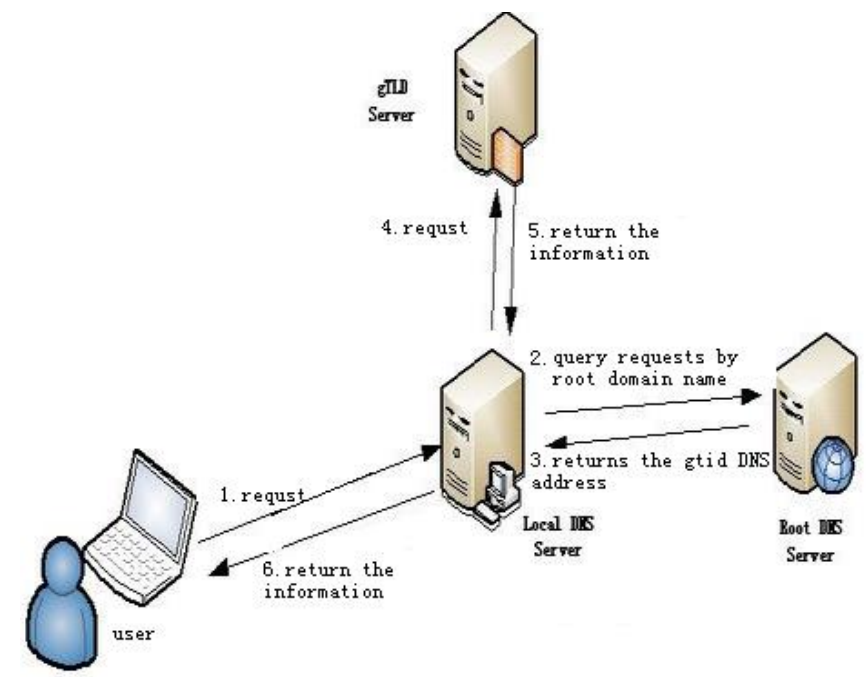

Figure 3. User Information Query Model

Such certification platform can realize the two-way real-name authentication, do not distinguish between the buyer or the seller as long as to trade on the net to be done realname authentication, both the seller is registered at the time of setting up shop, and the buyer need to be registered in the actual transactions. And the platform is based on DNS system, takes full advantage of the characteristics of the DNS system layered management, solves the data quantity problem online users unified management, can realize hierarchical management [7]. In addition, in the whole registration process no user id in the transfer of information, the user registered in the DNS server needs to provide the id card information, and this system is very reliable, since the id information is written to the DNS system and will never be transmitted on the Internet, to ensure the reliability of the user's private information, can break he buyer registered users worrying because of fear of personal information loss. The transaction process, will not do some change with current transaction process, and the registration process is no change, registration link just changes the location to the DNS servers and is no longer the site, it is no different for users won't be trouble, also solve the user feel trouble problem in particularly identities of the transaction process (user here refers to the buyer, because the sellers also need the 
certification under the current system when they are setting up shop). The user can through the DNS query all IP number binding with the user number, and then can query the user credit information to every website.

\section{Model Implementation}

The using process of the model made a few changes in the existing trade process, so don't bring existing each website redesign, and does not interfere with the user's trading habits, the following Figure 4 will show the use process of the model.

It's Visible in the flow chart of the model using, on site compare with the current system without any changes in the business, only need to register through the link to the corresponding DNS server to do register, only need to make a web site link, the link is completed without the user's own but the website, because the technology for website is very easy to implement, and for users need to know the site correspond to the DNS server is very difficult to increase the difficulty of online shopping, and only through the website link to get the trade website IP, and the IP is the only source getting the user's trade credit information on the all trading websites. In addition to the web site needs to change nothing, all operations will be done on the DNS server, the number of relative website the number of DNS server is much less, so the workload can be achieved.

For DNS servers changes mainly embodied in two places:

First, on the home page to add a function which online trading customer service function, the function has two child function the registered and query, through registration first transaction users registration, wrong operation register user can be handled. Through the query function to achieve the binding the new IP of old user and his user information, and the query foe trading website Ip under the user number, and to query the user credit information of each site.

Second, the platform of a job is to manage user information database, this is a big change for the existing DNS system, for this the model using the domain name and IP table, under it links in a Table for user name under all IP in this network segment, and the Table only records the user information for first trading real-name authentication of , namely if the user is not first trading the user information will not generate a data with a certain IP, but found the user's data in the Table recorded its first trading IP and the new trading website IP will be inserted into the Table as a data item. This data management can be done through the domain name and IP table, IP Table of a certain website, user Table under a IP, these three tables constitute three-dimensional data relationship (see Figure 5) [8], and has implied for such huge data management commendably.

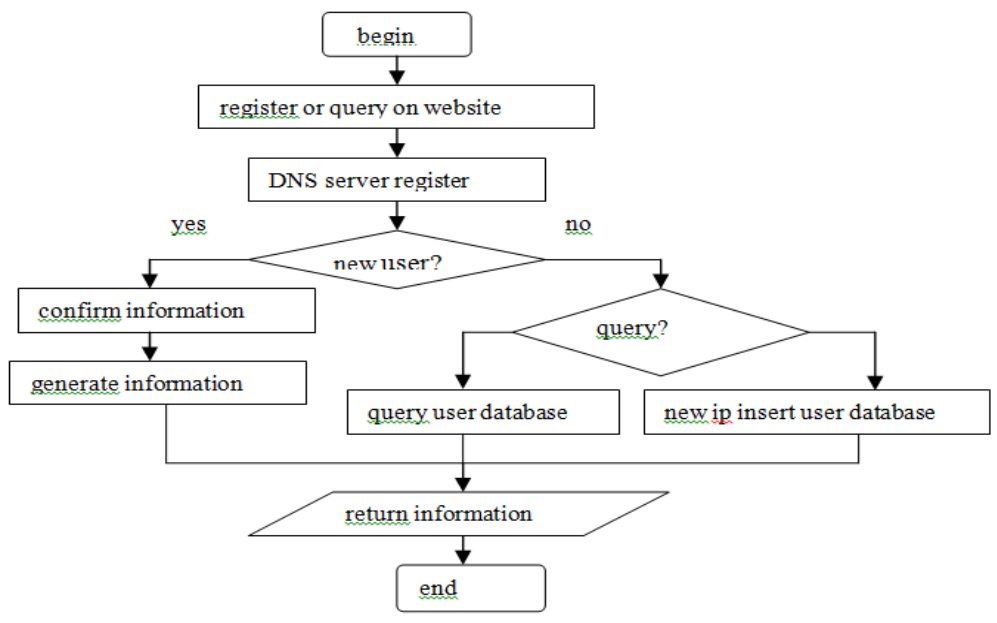

Figure 4. Model using Flow Chart 


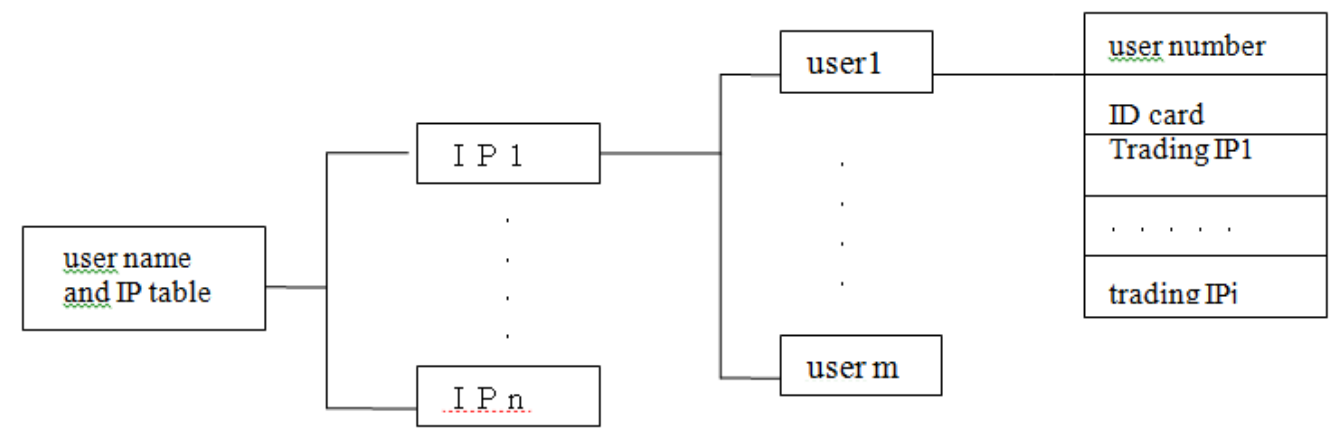

Figure 5. Three-dimensional Data Relationship Table

Here are the process of the users online trading by the platform :

(1) assume that the user i will carry on the online trading, at first it needs to the platform to register, when logins platform for registration system will detect the DNS his computer IP belongs to , and connect it to its own DNS server system.

(2) The system firstly show the query page, the user i input the ID number, as query results if is existing the user will be terminated entering the query page of user ID for registration, or to the registration page. (if it isn't queried in this DNS system of network segments at the corresponding level and then in accordance with the rules of DNS query system to a network segment to query at the next higher level of DNS query system, until the query to the root position, so the query result is not just a network segments at the corresponding level, if at some segment the user id information can be found then the same user ID is not registered repeatedly in the current DNS system the user belongs to, so avoids the possibility of a user through different IP to register, so registered user ID is still the only, to ensure the security of the scheme.)

(3) Entering the registration page user shall be carried out in accordance with the prompted to fill out the registration information, registers successfully will receive a user ID number, is the only password online trading and credit evaluation, assumes that the user $i$ gets a user id as ABCDEFGHIJKL. (at there the eight coded information, the first and the second are the state information, the third and fourth are region information, the fifth and the sixth are the information of the DNS system, the no seven to the no twelve are users' personal information, of course, the actual format of the coding subjects to specific statistics and investigation to determine.)

(4) Then the user can through the user ID to trade on the net, either for the buyer or for the seller management process is the same. Assumes that the user i made a deal with five users respectively in five sites, its every trade credit evaluation value is written into the various websites, but its each transaction website IP is written to the user management file of its user ID's DNS system users, if the user traded in the same site, the site will keep track of all of the user's credit evaluation results, and the platform will not repeat trading website IP information written to the DNS system, thereby reducing the useless data, optimize the resource management of the system. The user i information is shown in the Tablebelow:

\section{Table 1. User Information Yable}

\begin{tabular}{|l|l|l|l|l|l|l|}
\hline ABCDEFGHIJKL & 232403197705120730 & IP1 & IP2 & IP3 & IP4 & IP5 \\
\hline
\end{tabular}

(5) If the user $j$ want to query the credit value of user $i$ in some transaction, maybe login the platform to query at the corresponding DNS system according to the user ID information obtained in the transaction, by the user information Tablecan only see the IP address of the user transaction, can't see the user's ID card information, user $\mathrm{j}$ can query 
the user i credibility to each website based on IP trading website user. (every user will have two kinds of identities to be evaluated, namely the seller and the buyer in various web site)

(6) The platform provides user credit statistics, if users $\mathrm{j}$ hopes to get each site the composite index of the credit evaluation about user $i$, can provide platform with each website credit evaluation data (including the buyer and the seller two roles), the function of the platform to provide comprehensive statistics, at last, through the form of a graph to show the user comprehensive evaluation to change trend. (the seller and the buyer both as to the identity of the value will be shown in a picture, making it easy for users to compare, if the user has some kind of identity does not value the curve does not show in the graph.) As shown in Figure 6. The user can also choose time interval to query the credit value.

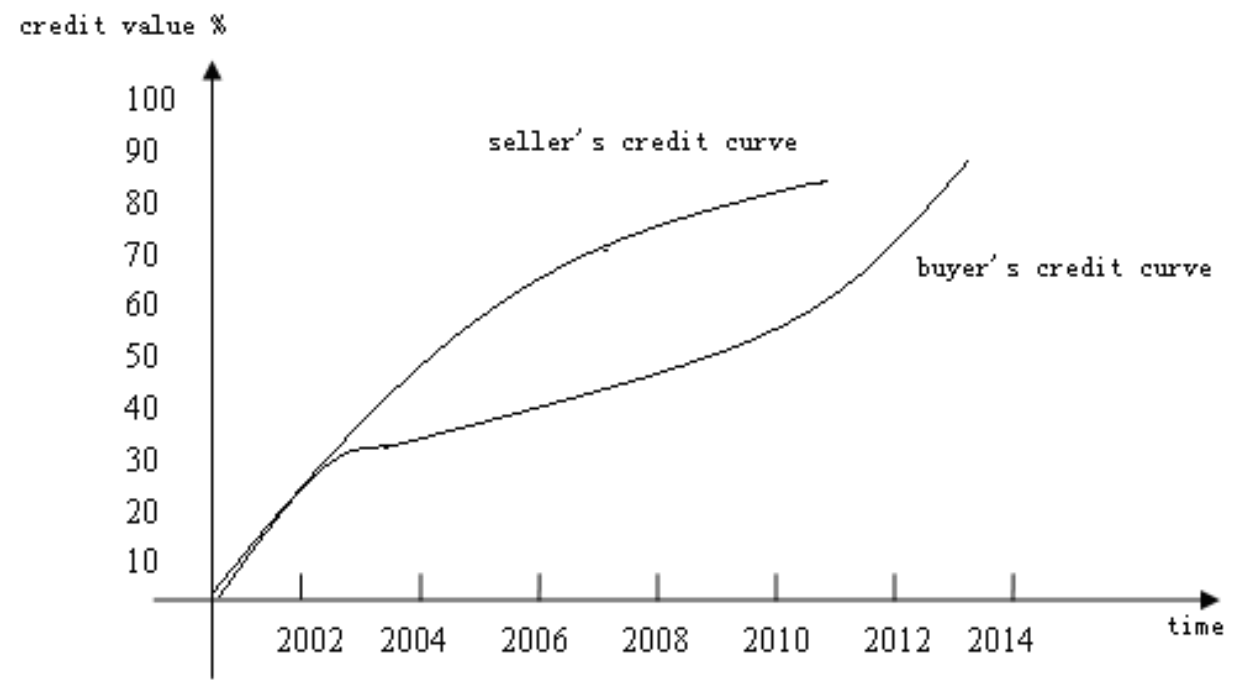

Figure 6. User Comprehensive Credit

\section{Others Suggestions}

The two-way real-name authentication unified platform based on DNS system designed in this paper has important practical significance, but proposed nature change to online transactions change, so need the support of the whole society in every way, at the same time due to want to make it as far as possible to adapt to the current online trading platform, so did some circumvent for these ideas, here it will expound some suggestions of this article did not mention here, hope to get everybody's attention and give guidance.

First, considering the various sites for competing want to protect their information well, user credit information is website's resources, so it is not willing to open, so this article only provides a platform for users to query trading website IP by user number by themselves, and then to query the user credit value on every trading website, this is more troublesome for the user, and the contrast is not intuitive. If every transaction credit value can be supported to the DNS system by website, and the credit information will be managed in DNS system, it can facilitate the user's query and compare.

Second, the real-name authentication is only about the uniqueness of ID card information but no authenticity, Users may use a fake id, or with other people's id card to register, such behavior has brought great harm to online trading of credit. This paper designs platform for the certification by one number management integrates the identity card number and user information, avoid the user with an id card registered in different sites and different identity and registered at the same site, but there is still no certification the reality of ID card, this problem can be solved by the networking the DNS system and the public security network. However, the public security network for high security requirements, will try to circumvent the open network, and the changes for the DNS 
system are increased, ultimately did not design this plan considering the difficulty of implementing in this article, but it is the root online information flood.

\title{
5. Conclusion
}

E-commerce is permeating every corner of people's life in rapid development momentum, can't say that e-commerce will replace traditional business market, but can say it is really seriously affecting the traditional market, Traditional markets in some areas can only reverse the role of online trading terminal service role, said that although not comprehensive, but to some extent, the traditional market is beginning to get used to this kind of role, visible electronic commerce future prospects beyond measure. Closest to the people's life is $\mathrm{C} 2 \mathrm{C}$ e-commerce, $\mathrm{C} 2 \mathrm{C}$ e-commerce's biggest problem is the credit problem, it is imperative to solve the problem! In this paper, based on DNS system of $\mathrm{C} 2 \mathrm{C}$ e-commerce two-way real-name authentication unified platform fundamentally can solve the $\mathrm{C} 2 \mathrm{C}$ e-commerce credit problems. Although there are a variety of difficulties in the implementation, is likely to be a great challenge to the existing system, but the road of growth never fears challenge, hope people from all walks of life to $\mathrm{C} 2 \mathrm{C}$ e-commerce credit problems give highly attention and support!

\section{References}

[1] P. Chunhui, A. Jing and F. Meiqi, "Credibility Evaluation Model and Arithmetic Study of C2C Ecommerce Site", Journal of Information, no. 8.

[2] J. F. Rayport and B. J. Jaworski, "Introduction to e-commerce", McGraw Hill, (2003).

[3] G. P. Schneider, "Electronic Commerce", Machine industry publisher, (2003).

[4] T. J. Strader and S. N. Ramaswami, "Trustworthiness in C2C Online Markets", Communications of the ACM, vol. 45, no. 12, (2002), pp. 87-102.

[5] Dellarocas, "The digitization of word of mouth Promise and challenges of online feedback mechanisms", Management Science, vol. 49, no. 10, (2003), pp. 1003-1057.

[6] W. Xiaoxia and B. Min, "The Credit Search of C2C E-commerce for Our Country", Decision and Information, no. 6, (2008).

[7] T. Junfeng and T. Rui, "A Fine-Grain Trust Model Based on Domain and Bayesian Network for P2P ECommerce System", Journal of Computer Research and Development, vol. 48, no. 6, (2011).

[8] T. Yongxin and L. Zengliang, "Progress in Software Trustworthiness Metrics Models", Computer Engineering and Applications, vol. 46, no. 2, (2010), pp. 12-16.

\begin{abstract}
Author
Xiaotang Li, In December 1979 was born in ZhaoDong city Heilongjiang province of china. Since 2002 has worked in computer science and Technology College of Harbin University of commerce, the research direction is electronic commerce.
\end{abstract}


International Journal of Security and Its Applications

Vol. 9, No. 5 (2015) 